We report on and discuss a case of a female patient diagnosed with breast cancer in 1996, which was histopathologically assessed as an invasive ductal carcinoma. The patient was admitted to our Department in 2017 with a liver metastasis of a neuroendocrine tumour. On admission she had no symptoms of an endocrinopathy and was in a good general condition. Due to unknown primary site of the metastasis and given the patient's history of breast cancer, it was suspected that the breast cancer was in fact a neuroendocrine tumour. This hypothesis was confirmed by comparing histopathological specimens of the breast and liver tumours using advanced pathological methods.

Key words: neuroendocrine tumour breast carcinoma, metastasis, liver.

Contemp Oncol (Pozn) 2018; 22 (2): 124-128 DOI: https://doi.org/10.5114/wo.2018.76831

\section{Liver metastasis}

of a neuroendocrine neoplasm of unknown primary origin in a female patient with a history of breast cancer

\author{
Małgorzata Struk-Panfil ${ }^{1}$, Krzysztof Błaut ${ }^{1}$, Rafał Pęksa ${ }^{2}$, Piotr Kmieć ${ }^{1}$, \\ Krzysztof Sworczak ${ }^{1}$
}

${ }^{1}$ Department of Endocrinology and Internal Medicine, Medical University of Gdansk, Gdansk, Poland

${ }^{2}$ Department of Pathology, Medical University of Gdansk, Gdansk, Poland

\section{Introduction}

Neuroendocrine neoplasms (NENs) are rare and often present with unspecific symptoms. In more than $10 \%$ of all NENs the primary origin is unknown. Establishing the primary location can be challenging, and the diagnostic work-up includes anamnesis, immunohistochemical examinations of metastatic lesions, and various imaging techniques, such as whole body CT, somatostatin receptor scintigraphy, and ${ }^{68} \mathrm{Gallium}$ - and ${ }^{18} \mathrm{~F}$-fluorodeoxyglucose positron emission tomography/computed tomography $\left({ }^{18} \mathrm{~F}-\mathrm{FDG} \mathrm{PET} /\right.$ CT) scans. In $70 \%$ of cases the primary neuroendocrine tumour occurs in either the gastrointestinal or pulmonary tract. However, there are also other, rare, primary locations: the genitourinary system (the uterine cervix, vagina, urinary bladder), the thymus, larynx, skin, and the breast.

\section{Case report}

A 50-year-old female, with a history of cancer of the left breast, treated more than 20 years earlier (by mastectomy, chemotherapy, radiotherapy, and hormone therapy), was admitted to the Department of Endocrinology and Internal Medicine of the Medical University of Gdańsk in October 2017 due to a liver metastasis of a neuroendocrine tumour of unknown origin.

As part of her oncological follow-up, in November 2016, a heterogeneous, polycyclic focal lesion was detected in the right lobe of the liver, measuring $45 \times 21 \times 29 \mathrm{~mm}$ in abdominal ultrasonography and, subsequently, confirmed in a whole-body CT scan. A metastasis was suspected; however, its primary origin was not revealed. The result of a fine-needle aspiration biopsy (performed in December 2016) was inconclusive.

In February 2017, the liver tumour was resected and histologically a neuroendocrine neoplasm metastasis was stated (NEN G2, immunohistochemistry: synaptophysin+, chromogranin+, Ki67 10\%).

In April 2017, in somatostatin receptor scintigraphy, another metastasis in a supradiaphragmatic lymph node was found, while in August 2017 a FDG-PET scan revealed intense tracer uptake in the thoracic and abdominal lymph nodes and in the right lobe of the liver.

In October 2017, the patient was admitted to our Department. On admission she was in a good general condition, without signs or symptoms of an endocrinopathy, and her body weight was stable. Laboratory tests did not reveal any hormonal activity of the tumour (concentrations of insulin, gastrin, calcitonin, and chromogranin A were within reference ranges; 24-hour 


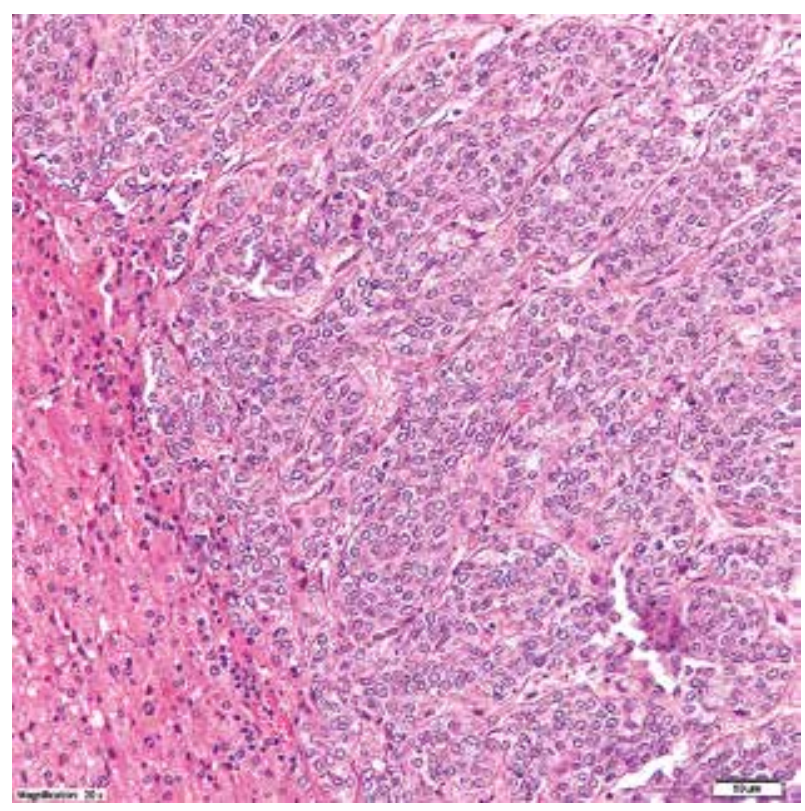

Fig. 3. Liver metastasis of the neuroendocrine tumor, composed by monomorphic cells arranged in trabeculae (H\&E, 20x)

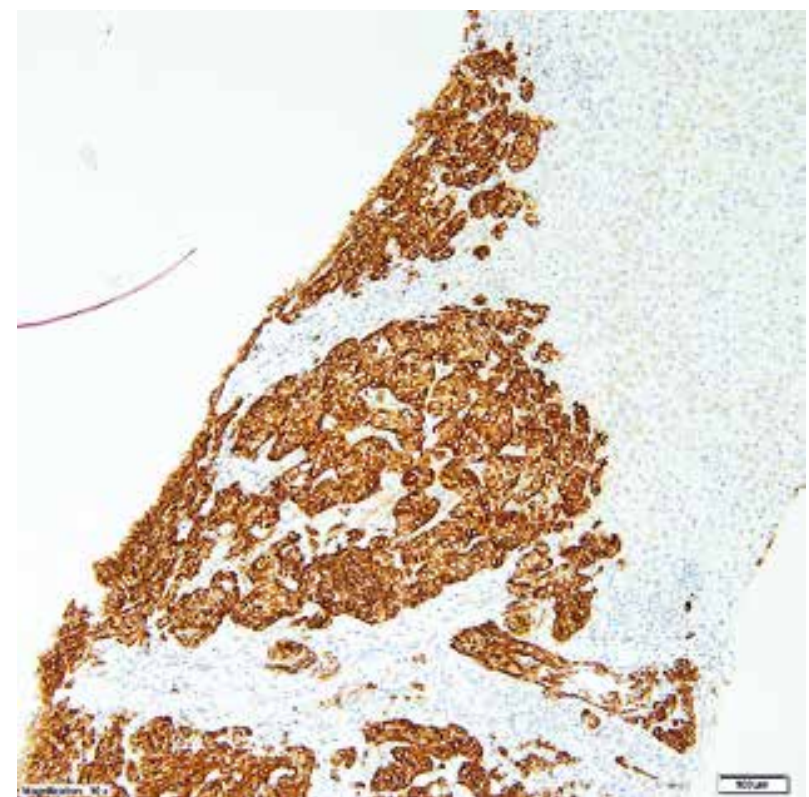

Fig. 5. Chromogranin A expression in cells of the metastatic tumour of the liver (20x)

In a retrospective review, most NECB were WD-NETs (55\%), followed by PD-NECs and IBC-NEDs [1]. The last group is underrecognised, due to varying inclusion criteria and lack of routine immunohistochemical staining for neuroendocrine markers.

Neuroendocrine carcinoma has no notable or specific clinical features distinct from other types of breast cancer: it may present as a hard breast lump with or without axillary lymphadenopathy. Very rarely, patients present with hormonal secretion symptoms (mainly ACTH, calcitonin, and norepinephrine) due to ectopic hormone production [3]. Definitive diagnosis is made by core needle biopsy or surgical specimens.

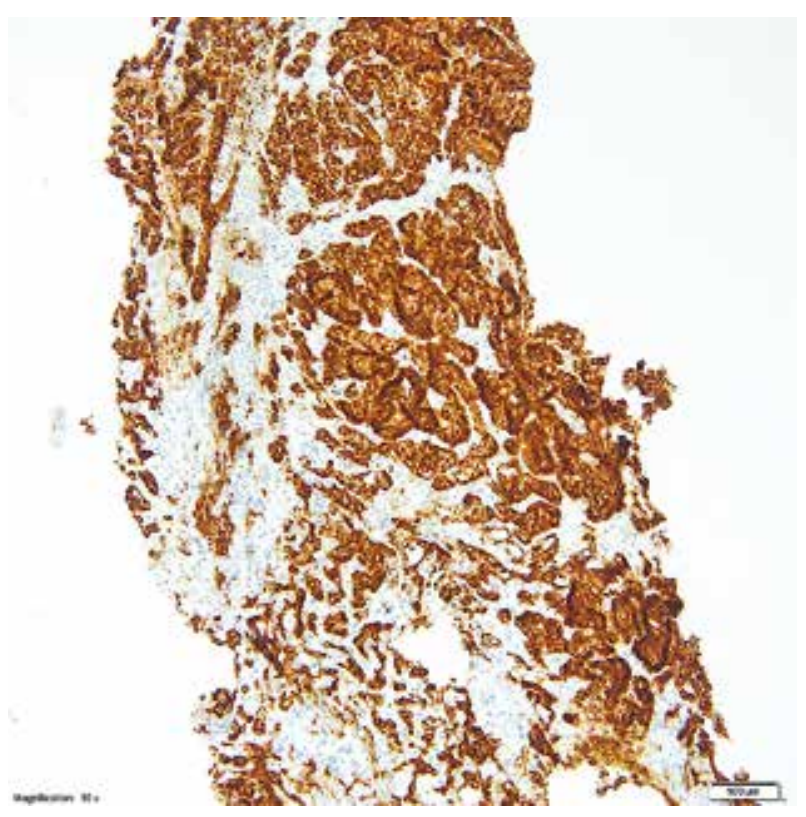

Fig. 4. Synaptophisin expression in cells of the metastatic tumour of the liver $(20 x)$

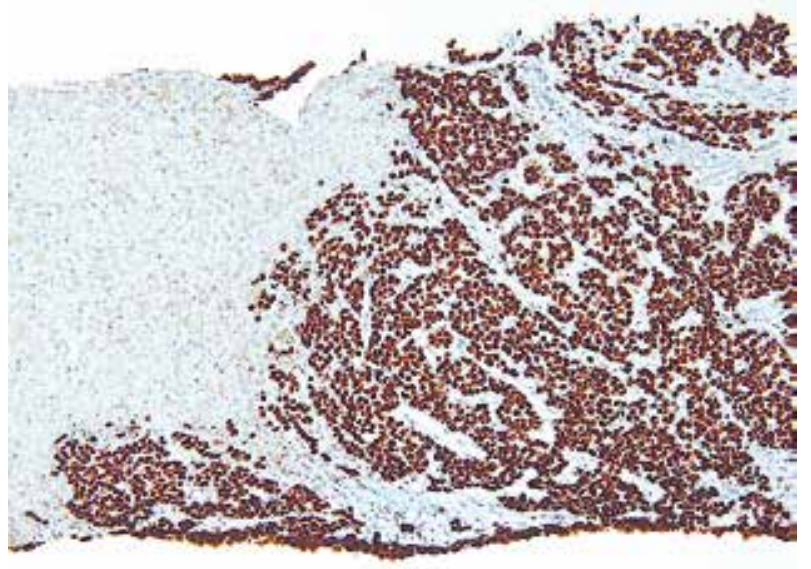

Fig. 6. GATA3 expression in cells of the metastatic tumour of the liver $(20 x)$

Establishing the diagnosis requires fulfilment of three criteria: revealing expression of neuroendocrine markers in more than $50 \%$ of the cell population (synaptophysin and/or chromogranin), clinical exclusion of metastatic neuroendocrine carcinoma, and histological demonstration of in situ tumour component within the breast [4].

Typical histological features that suggest endocrine differentiation include: uniform cells (round- or spindleshaped), nuclear palisading, abundant finely granular eosinophilic cytoplasm, and nuclei with "salt and pepper" chromatin. Tumour cells form nests, islands, and alveolar-like structures surrounded by delicate fibrovascular stroma. Although morphologic features may suggest neu- 


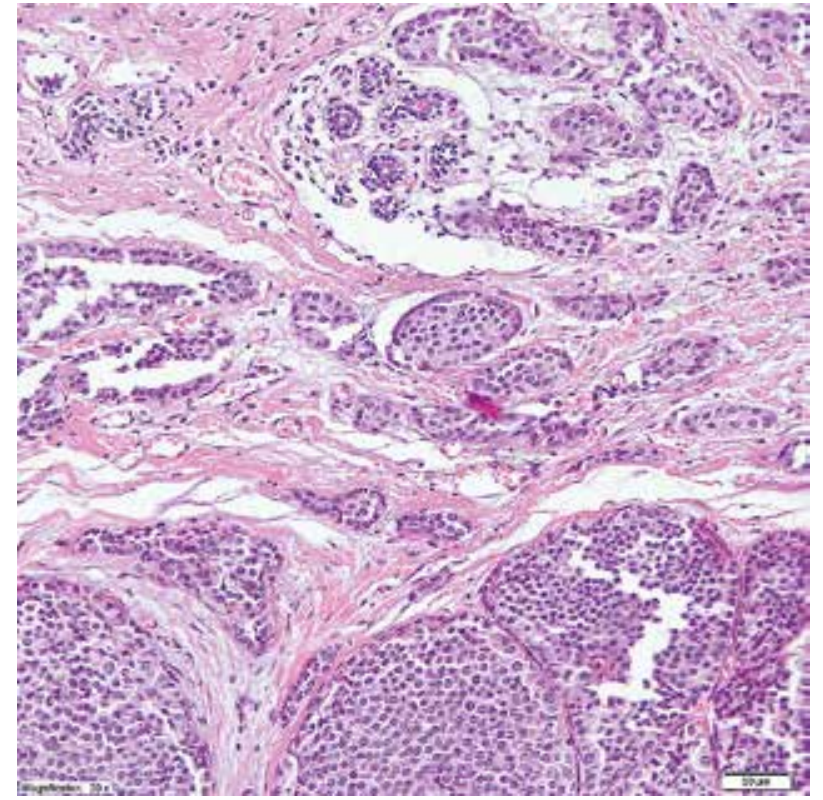

Fig. 1. Primary tumour of the breast formed by uniform cells with scant, lightly eosinophilic cytoplasm, arranged in broad gyriform trabeculae. Invasive component is visible at the top, in situ component below it (H\&E, 20x)

urinary excretion of 5-hydroxyindoloacetic acid was only slightly above the upper reference value); neuron-specific enolase (NSE) concentration was elevated.

Due to her relatively young age, no primary site of NEN metastases, and history of breast cancer (diagnosed in 1996), neuroendocrine breast cancer metastases discovered 21 years after the primary tumour treatment were suspected. This hypothesis was confirmed by comparing histopathological specimens of breast and liver tumours (from 1996 and 2017, respectively). Histopathological examination of the breast tumour specimen confirmed a primary neuroendocrine carcinoma of the breast (a well-differentiated neuroendocrine neoplasm - WD-NEN) with vascular invasion (Fig. 1) and the following immunophenotypes: synaptophysin+ (Fig. 2), chromogranin+, Ki67 3\%, ER: $60 \%$, PR $80 \%$, and HER2-0. The liver tumour specimen also revealed a neuroendocrine tumour (Fig. 3) with the following immunohistochemical characteristics: synaptophysin+ (Fig. 4), chromogranin+ (Fig. 5), GATA3+ (Fig. 6), HepPar1-, Glypican3-, Ki67 of 12\%, ER 40\% (Fig. 7), PR 0\%, and HER2-0. The final diagnosis in this patient was: a disseminated neuroendocrine neoplasm of the breast with metastases to the liver and lymph nodes of the thorax and abdomen.

The patient was referred for further treatment, i.e. thermoablation of hepatic lesions. Peptide receptor radionuclide therapy was planned as the consecutive stage of treatment.

\section{Discussion}

Primary neuroendocrine carcinoma of the breast (NECB) is a rare and underrecognised subtype of the mammary carcinoma [1]. It is considered to account for approximately $1 \%$ of all breast cancers and $1 \%$ of all neuroendocrine

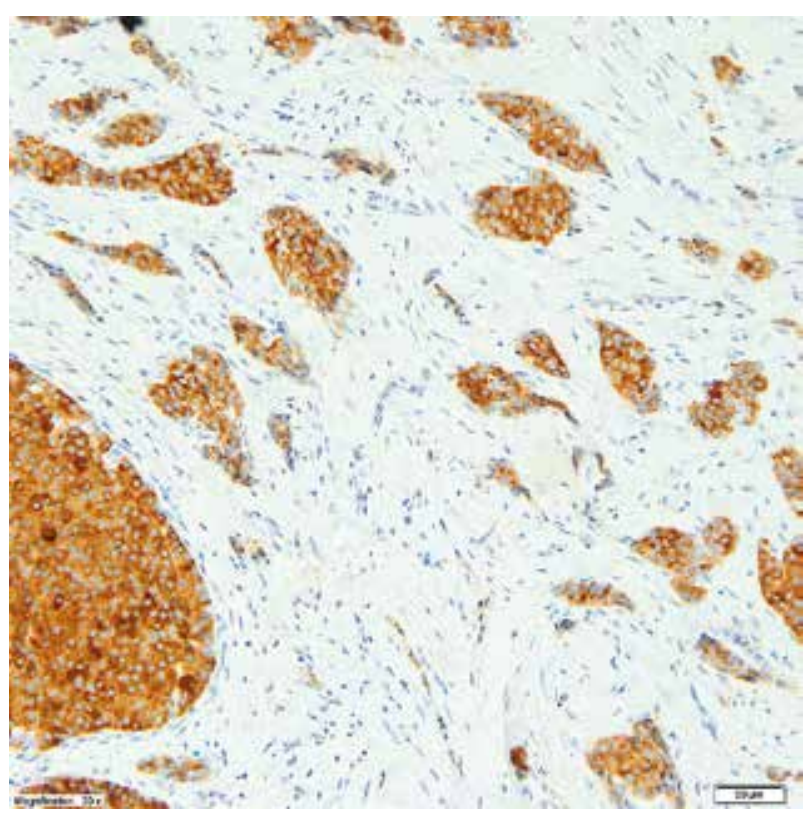

Fig. 2. Synaptophisin expression in cells of the primary tumour of the breast $(20 x)$

tumours. It is possible its incidence is underestimated because neuroendocrine markers are still not routinely used in the diagnostic work up of breast cancer cases.

Based on available medical literature including clinical case reports, it is believed that NECB occurs predominantly in white postmenopausal women in the sixth and seventh decades of life [2]. However, as our case demonstrates, neuroendocrine breast cancer may also occur in the third decade of life.

The histogenesis of NECB is still debated. The most prevalent and accepted hypothesis is that neuroendocrine cells arise during early carcinogenesis of neoplastic stem cells into epithelial and endocrine cells. This theory is supported by the lack of neuroendocrine cells in benign tumours of the breast, and the fact that NECB always comprises both endo- and exocrine cells [3].

Neuroendocrine carcinoma of the breast was recognised as a separate entity by World Health Organisation (WHO) in 2003. In 2012, the WHO established a new classification and definition of NECB; it was acknowledged that for this diagnosis the $50 \%$ threshold of neuroendocrine marker expression in tumour cell populations was obligatory; however, the classification also included "invasive breast carcinoma with neuroendocrine differentiation" (IBC-NED), regardless of the percentage of tumour cells expressing neuroendocrine markers.

According to the current WHO classification, NECB are subclassified into: a) well-differentiated neuroendocrine carcinoma (WD-NEC) which has low- to intermediate-grade nuclear features and is similar to other neuroendocrine neoplasms of the gastrointestinal tract and the lung, b) poorly-differentiated neuroendocrine carcinoma or small cell carcinoma (PD-NEC), which is similar to small cell lung cancer, and c) IBC-NED [4]. 
roendocrine differentiation, the diagnosis of a neuroendocrine tumour of the breast requires demonstration of the expression of neuroendocrine markers $[5,6]$.

First-line markers of neuroendocrine differentiation are chromogranin A, synaptophysin, and CD56. CD56 is the most sensitive, but it is not entirely specific. A lowgrade neuroendocrine neoplasm (i.e. WD-NET) expresses chromogranin, synaptophysin, as well as CD56. However, staining in high-grade neuroendocrine neoplasm (i.e. small cell carcinoma) may be negative for chromogranin A and synaptophysin but positive for CD56. Neuron-specific enolase (NSE) is also used, but its specificity is lower [6]. In addition, most NECBs are progesterone and oestrogen receptor positive and HER2 negative.

Becuase NECB is a very rare entity, metastasis of a neuroendocrine tumour from the gastrointestinal tract, pancreas, or lungs should first be excluded. It may be challenging, when no primary origin is revealed in CT-scans and somatostatin receptor scintigraphy in those locations. Moreover, ER and PR are not specific immunohistochemical markers of cancers of mammary origin. Although ER and PR are quite frequent in NECBs (positive in the majority of tumour cells in WD-NETs, and > 50\% of small cell/ poorly differentiated carcinomas), they can also be positive in GEP-NETs or primary pulmonary NETs [7, 8]. GATA3 is a very useful marker, which can confirm the breast as the primary origin of neoplasm metastases. It is a transcription factor involved in the differentiation of breast epithelium, uroepithelium, and a subset of T-lymphocytes. GATA3 is considered one of the most specific markers for the breast as the origin of a metastatic carcinoma: it was detected in $92 \%$ of primary ductal carcinomas of the breast and $96 \%$ of breast cancer metastases [9].

Due to its rare incidence, prognosis of NECB patients compared to those with other types of breast cancer is still uncertain. Most studies indicate that NECB is an aggressive neoplasm. Higher grade, tumour size, and metastases to lymph nodes are associated with a poorer prognosis (decreased disease-free survival). However, mucinous differentiation and expression of ER and PR are favourable prognostic factors. In most studies, NECBs have a significantly higher rate of local and distant recurrence than IDC-NOS [1]. NECB can metastasise to multiple sites, even many years after treatment of the primary tumour; therefore, long-term follow-up is recommended. Most often, metastases are found in the lymph nodes, liver, bones, lungs, pancreas, soft tissues, and the brain [10].

\section{Conclusions}

There are no specific recommendations for the treatment of NECB. Usually, the same therapeutic strategy is applied as for other types of invasive breast carcinoma, not otherwise specified (NOS) [6].

The mainstay of therapy, depending on tumour location and stage, is surgical management. There are no specific studies concerning adjuvant radiotherapy in NECB, but it should be considered, similarly to other invasive breast cancers. Due to biological features of the disease, and the risk of recurrence, chemotherapy should be considered. It

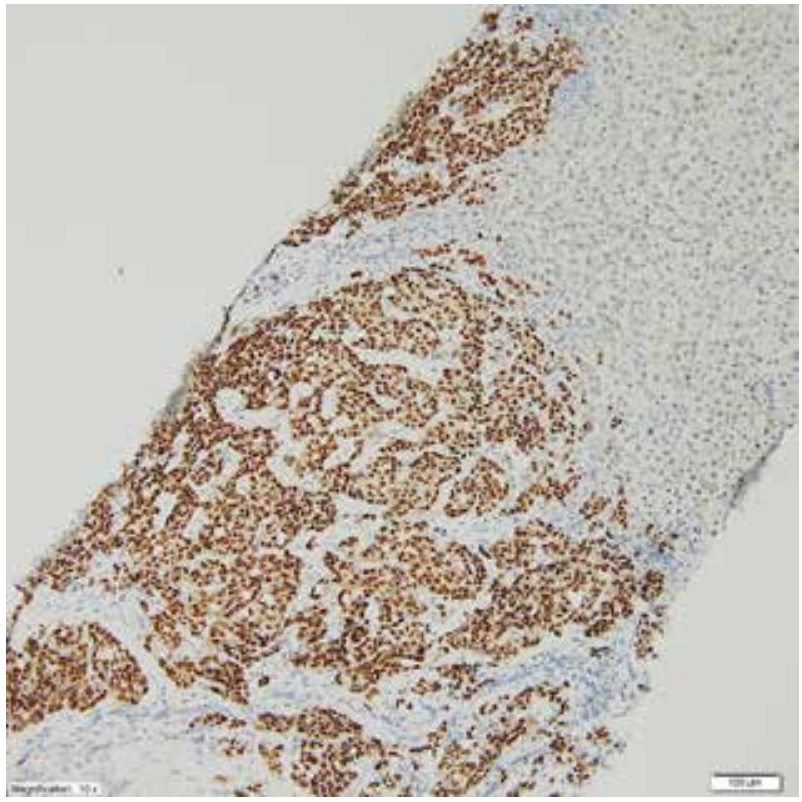

Fig. 7. ER expression in cells of the metastatic tumour of the liver $(20 \times)$

can be used in patients with a high risk of relapse (indicated by tumour size and nodal metastases) or in neoadjuvant therapy of inoperable or locally advanced NECBs. Patients with hormone-receptor positive NECBs are candidates of endocrine therapy [3].

If metastases are diagnosed, the treatment strategy is determined individually depending on the patient's condition, comorbidities, and malignancy of the tumour. Metastasectomy is an important therapeutic option [3].

Resection of a liver metastasis should be considered, if it is possible to remove it completely (RO) and there are no extrahepatic foci (with the exception of bone metastases that can be controlled by radiotherapy) [3]. According to some reports, hepatectomy may prolong the survival of patients with NECB [10]. Stereotactic radiotherapy is an alternative to surgery. In the case of multiple metastases, systemic treatment should be considered [3].

In patients with NECB positive in somatostatin receptor scintigraphy or ${ }^{68} \mathrm{Gallium}$ PET-CT scans, peptide receptor radionuclide therapy (PRRT) should be considered. Some authors reported that it was well tolerated and yielded good results. PRRT can be applied as first- or second-line therapy and for cases in which conventional chemotherapy is ineffective [3].

The authors declare no conflict of interest.

\section{References}

1. Rosen LE, Gattuso P. Neuroendocrine Tumors of the Breast. Arch Pathol Lab Med 2017; 141: 1577-1581.

2. Murthy VS, Geethamala K, Kumar BD, Sudharao M. Primary Neuroendocrine Carcinoma of Breast: A Rare Case Report. Ann Med Health Sci Res 2017; 3 (Suppl. 1): S35-S37. 
3. Inno A, Bogina G, Turazza M, et al.; Neuroendocrine Carcinoma of the Breast: Current Evidence and Future Perspectives. The Oncologist 2016; 21: 28-32.

4. Marinova L, Malinova D, Vicheva S. Primary Neuroendocrine Carcinoma of the Breast: Histopathological Criteria, Prognostic Fac tors, and Review of the Literature. Case Rep Pathol 2016; 2016: 6762085

5. Rosen PP. Mammary carcinoma with endocrine features. Breast Pathology. 2nd ed. Lippincott Williams \& Wilkins. Philadelphia 2001: 503-508

6. Lloyd RV. Practical markers used in the diagnosis of neuroendocrine tumors. Endocr Pathol 2003; 14: 293-301.

7. Sica G, Wagner PL, Altorki N, Port J, Lee PC, Vazquez MF, Saqi A. Immunohistochemical expression of estrogen and progesterone receptors in primary pulmonary tumours. Arch Pathol Lab Med 2008; 132: 1889-1895

8. Zimmermann N, Lazar-Karsten P, Keck T, Billmann F, Schmid S, Brabant $G$, Thorns $C$. Expression pattern of CDX2, estrogen and pro geserone receptors in primary gastroenteropancreatic neuroendo crine tumours and metastases. Anticancer Res 2016; 36: 921-924.

9. Miettinen M, McCue PA, Sarlomo-Rikala M, et al. GATA3: a multispecific but potentially useful marker in surgical pathology: a systematic analysis of 2500 epithelial and nonepithelial tumors. Am J Surg Pathol 2014; 38: 13-22.

10. Manes K, Delis S, Papaspyroua N, Ghiconti I, Dervenis C. Neuroen docrine breast carcinoma metastatic to the liver: Report of a case and review of the literature. Int J Surg Case Rep 2014; 5: 540-543.

\section{Address for correspondence}

\section{Małgorzata Struk-Panfil}

Department of Endocrinology and Internal Medicine

Medical University of Gdansk

3a M. Skłodowskiej-Curie St.

80-210 Gdansk, Poland

e-mail: malgorzata.struk@gmail.com

Submitted: 26.04 .2018

Accepted: 27.05 .2018 